\title{
ABORDAJE ORBITARIO PARA EL TRATAMIENTO DE LA FÍSTULA CARÓTIDO-CAVERNOSA
}

\author{
ORBITARY APPROACH FOR TREATMENT OF CAROTID- \\ CAVERNOUS SINUS FISTULA
}

\author{
MESA JC ${ }^{1}$, MASCARÓ $\mathrm{F}^{2}$, MUÑOZ $\mathrm{S}^{2}$, PRAT J ${ }^{3}$, ARRUGA J ${ }^{1}$
}

\begin{abstract}
RESUMEN
Caso clínico: Varón con fístula carótido-cavernosa (FCC) izquierda y antecedentes previos de embolización no exitosos del seno petroso inferior izquierdo con cateterismo venoso femoral. Se realizó embolización de la fístula canalizando directamente la vena oftálmica superior (VOS).

Discusión: La principal modalidad de tratamiento para las FCC es la radiología intervencionista que pretende la oclusión de la fístula vía arterial a través del seno cavernoso; o por vía venosa, a través del seno petroso inferior. Sin embargo, también puede accederse a la FCC a través de la VOS.
\end{abstract}

Palabras clave: Fístula carótido-cavernosa dural, tratamiento endovascular, embolización, coils desprendibles.

\begin{abstract}
Case report: A male patient with a left carotidcavernous sinus fistula (CCSF) and two previous attempts of embolization via the femoral vein was treated with embolization through the superior ophthalmic vein (SOV).

Discussion: The main modality of treatment for CCSF is intervention radiology. This aims to occlude the fistula via an arterial route, through the cavernous sinus, or via a venous route, through the inferior petrosus sinus. However, the CCSF is also accessible through the SOV (Arch Soc Esp Oftalmol 2008; 83: 719-722).
\end{abstract}

Key words: Dural carotid-cavernous sinus fistula, endovascular treatment, embolization, detachable coils.

\section{INTRODUCCIÓN}

Se presenta el caso de un paciente varón de 81 años con una fístula carótido-cavernosa (FCC) indirecta. La embolización transfemoral puede resultar en una trombosis incompleta de la fístula.
Se puede canalizar la vena oftálmica superior (VOS) y cateterizarla retrógradamente para permitir el depósito de coils desprendibles en la fístula por medio de un microcatéter. La técnica es segura y efectiva cuando se lleva a cabo por un equipo multidisciplinario.

\footnotetext{
Recibido: 5/11/06. Aceptado: 27/10/08.

Servicio de Oftalmología. Hospital Universitari Bellvitge. Barcelona. España.

1 Doctor en Medicina.

2 Licenciado en Medicina.

3 Doctor en Medicina. Hospital Sant Joan de Déu. Barcelona. España.

Correspondencia:

J.C. Mesa

Hospital Universitari Bellvitge

Feixa Llarga, s/n

08907 Hospitalet de Llobregat (Barcelona)

España

E-mail: jcarlosmesa@mixmail.com
} 


\section{CASO CLÍNICO}

Varón de 81 años de edad con antecedentes de FCC en ojo izquierdo (OI) que presentó aumento de su proptosis habitual, dolor, edema y equimosis palpebral. En los 3 años anteriores se habían efectuado dos intentos de embolización mediante cateterismo venoso femoral e hipotensión controlada con recidiva del cuadro por lo que se indicó la embolización de la fístula canalizando directamente la VOS izquierda. La agudeza visual con corrección (AVcc) era 0,9 en ojo derecho (OD) y 0,3 en OI, y la tensión por aplanamiento de OD $16 \mathrm{mmHg}$ y de OI 23 $\mathrm{mmHg}$. En la biomicroscopía se observó quémosis conjuntival moderada, así como tortuosidad vascular conjuntival y episcleral en forma de «cabeza de medusa» de predominio en conjuntiva bulbar inferior (fig. 1). La gonioscopía con lente de Goldmann reveló un ángulo 4 (Shaffer) normal.

El examen de motilidad ocular demostró restricción de los movimientos en musculatura extrínseca en OI sin diplopía, y la exoftalmometría Hertel reveló una asimetría entre ambos globos oculares de $4 \mathrm{~mm}$ (OD 14/OI 18).

En la oftalmoscopía indirecta se observó una excavación fisiológica normal de la papila, relación arteria-vena $2 / 3$, así como tortuosidad e ingurgitación venosa. El examen del campo visual (perimetría automatizada Goldmann) mostró un aumento de la mancha ciega en OI.

La tomografía computerizada (TC) y resonancia magnética nuclear (RMN) orbitaria objetivaron la dilatación de la VOS izquierda (fig. 2) La arteriografía selectiva por vía femoral confirmó la existencia de una FCC izquierda tipo D de Barrow (fig. 3).

Se canalizó la VOS izquierda introduciendo un microcatéter hasta el seno cavernoso (SC). Se colocaron múltiples coils comprobando el aporte hacia la fístula desde la arteria carótida y observando el

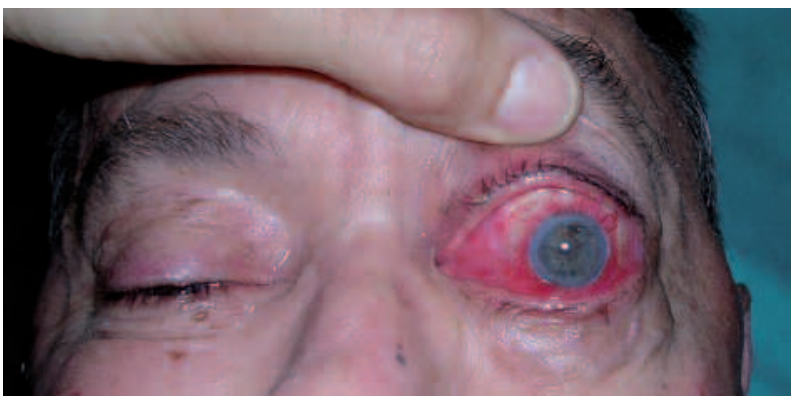

Fig. 1: Quémosis y exoftalmos ojo izquierdo.
SC excluido de la circulación así como la trombosis de la fístula (figs. 4 y 5).

Al mes de la intervención la exploración del paciente fue la siguiente: AVcc: OD: 0,9; OI: 0,5, presión intraocular (PIO) $16 \mathrm{mmHg}$ (ambos ojos). No se observaron alteraciones de la motilidad ocular, y la exoftalmometría objetivó una diferencia entre ambos globos oculares de $2 \mathrm{~mm}$ (14 OD /16 OI). El examen con lámpara de hendidura mostró una dilatación residual de vasos conjuntivales inferiores; el examen de fondo de ojo constató la desaparición de la tortuosidad vascular preexistente (fig. 6).

\section{DISCUSIÓN}

La FCC es una comunicación anómala entre el seno cavernoso y las ramas arteriales derivadas de la arteria carótida intracraneal. El 75\% tienen un origen traumático, por traumatismos cráneo-faciales o tras cirugía. El 25\% restante se origina de forma espontánea, siendo la arteriosclerosis un factor importante $(1,2)$. La clasificación arteriográfica las divide en directas (carótido-cavernosas) o durales (carótida externa). Barrow (1985) las clasifica en (3):

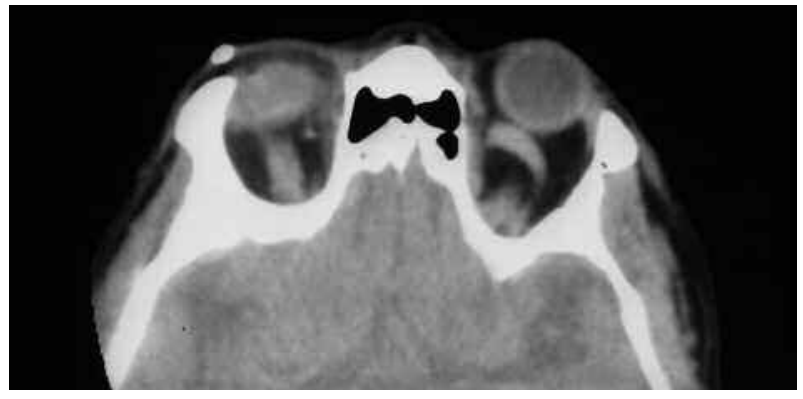

Fig. 2: Tomografía axial computarizada de órbita: dilatación y acodamiento de la vena oftálmica superior.

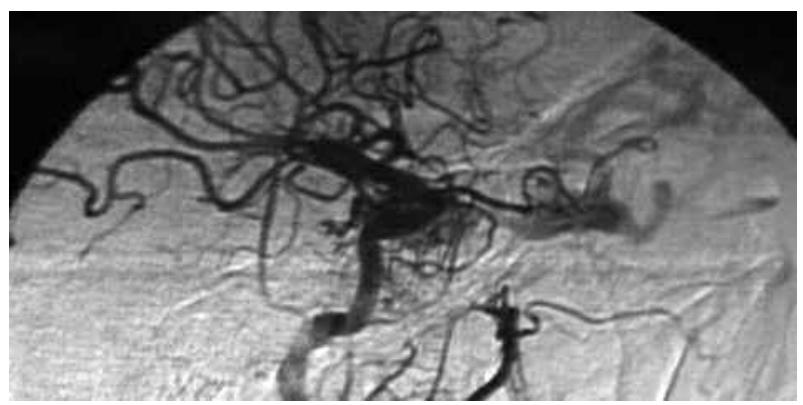

Fig. 3: Angiografía carótida izquierda: Relleno de seno cavernoso y vena oftálmica superior izquierdos. 


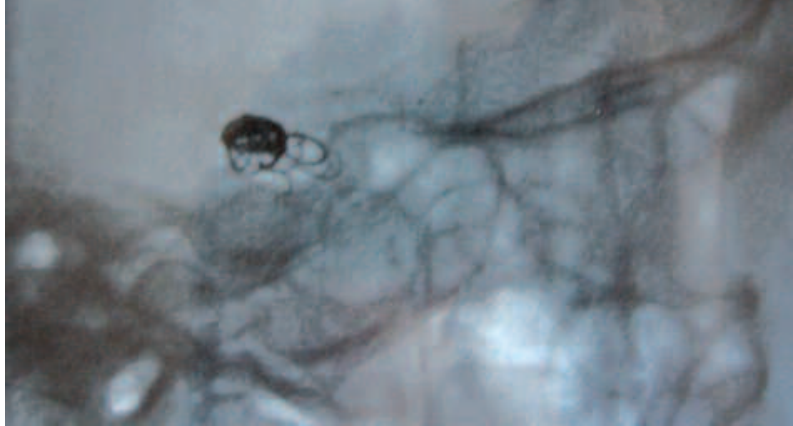

Fig. 4: Arteriografía vía femoral: Comprobación de coils en seno cavernoso.

- Tipo A, comunicación entre la arteria carótida interna (ACI) intracavernosa directamente con el SC. Es fundamentalmente traumática y de alto flujo.

- Tipo B, comunicación entre la ACI extracavernosa y el SC.

- Tipo C, comunicación entre ramas meníngeas de la arteria carótida externa (ACE) y el SC.

- Tipo D, comunicación entre ramas de la ACE y ACI con el SC.

Los tipos B, C y D suelen ser fístulas indirectas de bajo flujo, localizadas en los vasos de la dura que se presentan con sintomatología menos florida. Frecuentemente evolucionan a cierre espontáneo $(1,2,5)$.

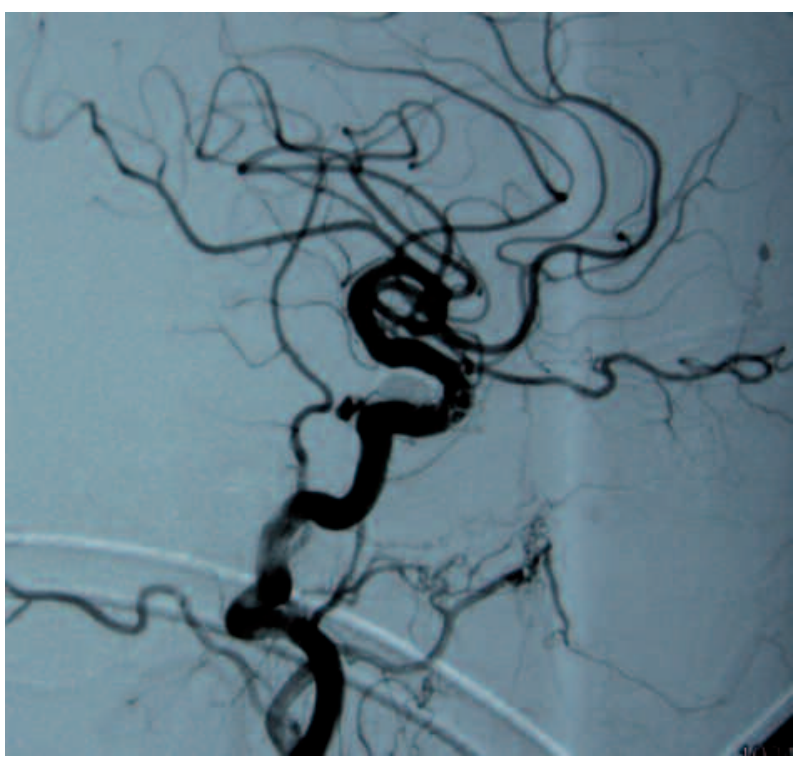

Fig. 5: Arteriografía vía femoral: comprobación cierre de FCC.

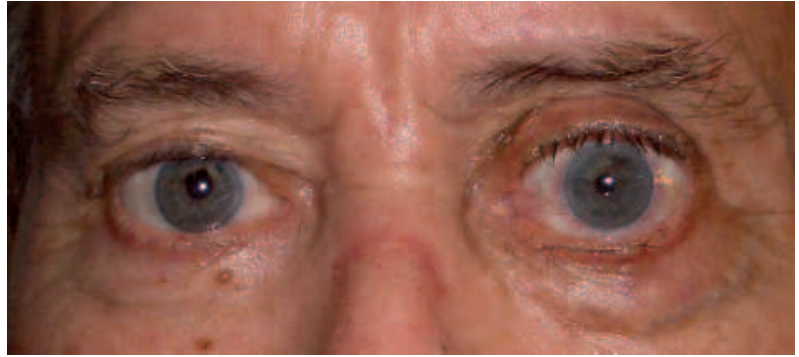

Fig. 6: Aspecto post-operatorio al mes del cierre de la fístula carótido-cavernosa.

En las FCC de bajo flujo se adopta una actitud terapéutica conservadora (compresiones carotídeas, hipotensores oculares) y seguimiento a largo plazo ya que entre el 25 y $50 \%$ se resuelven espontáneamente $(1,2,4,5)$. En las de alto flujo, en líneas generales, el tratamiento endovascular suele ser necesario para ocluir la fístula $(2,4,5)$.

Las siguientes condiciones precisan tratamiento quirúrgico (1):

1. Pérdida visual. El aumento de presión en el sistema venoso retiniano disminuye la presión de perfusión arterial, manifestándose como isquemia coroidea o retiniana.

2. PIO incontrolable con tratamiento médico máximo. La PIO aumenta por hipertensión venosa o por el desarrollo de neovascularización iridiana y cierre angular pretrabecular.

3. Cefalea intolerable.

4. Proptosis maligna con exposición corneal.

La embolización del SC puede ser por la vena femoral, yugular interna, VOS o vena oftálmica inferior $(2,4,5)$.

El porcentaje de éxito en el tratamiento de las FCC directas, entendiendo por tal la resolución de los síntomas clínicos con desaparición angiográfica completa o parcial de la fístula, oscila entre el 58 y $100 \%$ en las diferentes publicaciones $(1,2,4,5)$.

\section{BIBLIOGRAFÍA}

1. Burgueño Montañés C, Barbón García JJ, Muro Plaza M, Fernández Diego JI. Exoftalmos bilateral secundario a fístula carótido-cavernosa derecha. Arch Soc Esp Oftalmol. 2001; 76: 441-444.

2. Kirsh M, Henkes H, Leibig T, Weber W, Esser J, Golk S, et al. Endovascular management of dural carotid-cavernous sinus fistulas in 141 patients. Neuroradiology 2006; 48: 486-490. 
3. Barrow DL, Spector RH, Braun IF, Landman JA, Tindall $S C$, Tindall GT. Classification and treatment of spontaneous carotid-cavernous sinus fistulas. J Neurosurg 1985; 62: 248-256.

4. Baldauf J, Spuler A, Hoch HH, Molsen HP, Kiwit JC, Synowitz M. Embolization of indirect carotid-cavernous sinus fistulas using the superior ophthalmic vein approach. Acta Neurol Scand 2004; 110: 200-204.

5. Meyers PM, Halbach VV, Dowd CF, Lempert TE, Malek AM, Phatouros CC, et al. Dural Carotid cavernous fistula: definitive endovascular management and long-term follow up. Am J Ophthalmol 2002; 134: 85-92. 